\title{
Prognostic impacts and dynamic changes of cohesin complex gene mutations in de novo acute myeloid leukemia
}

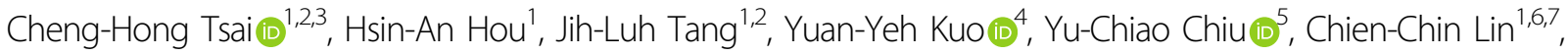 \\ Chieh-Yu Liư ${ }^{8}$, Mei-Hsuan Tseng ${ }^{1}$, Tzung-Yi Lin ${ }^{1}$, Ming-Chih Liu', Chia-Wen Liu', Liang-In Lin ${ }^{10}$, Ming Yao', \\ Chi-Cheng Li ${ }^{1,2}$, Shang-Yi Huang ${ }^{1}$, Bor-Sheng Ko ${ }^{1}$, Szu-Chun Hsu ${ }^{1,6}$, Chien-Ting Lin ${ }^{1,2}$, Shang-Ju Wu', \\ Chien-Yuan Chen', Woei Tsay ${ }^{1}$, Eric Y. Chuang ${ }^{5,11}$, Wen-Chien Chou ${ }^{1,6}$ and Hwei-Fang Tien ${ }^{1}$
}

Cohesin complex is a multimeric protein complex, composed of four core subunits, including SMC1A, SMC3, RAD21, and either STAG1 or STAG2 proteins. They form a ring-shaped structure, and mediate sister chromatid cohesion and segregation during mitosis and meiosis. Recently, the cohesin gene mutations have been reported in myeloid neoplasms ${ }^{1-7}$, but studies regarding their clinical and prognostic relevance and dynamic changes in de novo acute myeloid leukemia (AML) patients are limited and the findings are controversial.

In this study, we aimed to investigate the clinical, biological, and prognostic implications of cohesin gene mutations in a large cohort of de novo AML patients. To evaluate the sequential changes of cohesin and cooccurring gene mutations, serial analyses of gene mutations by targeted next-generation sequencing (NGS) were performed in 386 samples from 116 patients during follow-ups. To the best of our knowledge, this is the first report to address the dynamic changes of cohesin gene mutations during the clinical course in de novo AML. We also investigated the pathophysiological pathways by mRNA expression profiling.

A total of 391 consecutive patients with newly diagnosed de novo non-M3 AML, consisting of 217 males and 174 females, were recruited. The coding sequences of cohesin complex genes were screened by Ion Torrent

Correspondence: Wen-Chien Chou (wchou@ntu.edu.tw) or Hwei-Fang Tien (hftien@ntu.edu.tw)

${ }^{1}$ Division of Hematology, Department of Internal Medicine, National Taiwan University Hospital, Taipei, Taiwan

${ }^{2}$ Tai-Cheng Stem Cell Therapy Center, National Taiwan University, Taipei,

Taiwan

Full list of author information is available at the end of the article
NGS (Thermo Fisher Scientific, MA, USA). All mutations were confirmed by Sanger sequencing. For nonsynonymous missense mutations, we included only those reported to be pathogenic in literature, but not those predicted to be pathogenic solely by computational tools. Thirty-seven patients $(9.5 \%)$ had cohesin gene mutations, most commonly in RAD21 (15 of 391, 3.8\%), followed by STAG2 (12 of 390, 3.1\%) and SMC1A (8 of 391, 2.0\%). Except for one patient with concurrent mutations in STAG2 and RAD21, the mutations in these component genes were mutually exclusive, suggesting a convergence of biological effects of these mutations (Fig. 1a). Mutations in STAG2 and RAD21 were mainly truncations or frameshift mutations (10/12 and 12/15, respectively), while those in $S M C 1 A$ and $S M C 3$ were mostly missense mutations $(7 / 8$ and $2 / 2$, respectively; Fig. 1b).

Cohesin gene mutations were mutually exclusive with unfavorable-risk cytogenetics as well as complex chromosomal changes $(P=0.003$ and $P=0.023$, respectively, Supplementary Table S1), against that cohesin gene mutations lead to premature sister chromatid separation in AML. Therefore, cohesin gene mutations may take part in leukemogenesis by alternative mechanisms. Interestingly, six $(16.7 \%)$ of the thirty-eight patients with $t(8 ; 21)$ had cohesin gene mutations, all in $R A D 21$, while none of the patients with inv(16) had any cohesin mutation, compatible with previous reports ${ }^{1,8}$, indicating that concerted interaction of cohesin gene mutations with RUNX1-RUX1T1 fusion plays a role in the leukemogenesis of some AML patients with $t(8 ; 21)$. In the 34 patients with acute promyelocytic leukemia and $t(15 ; 17)$ who were 


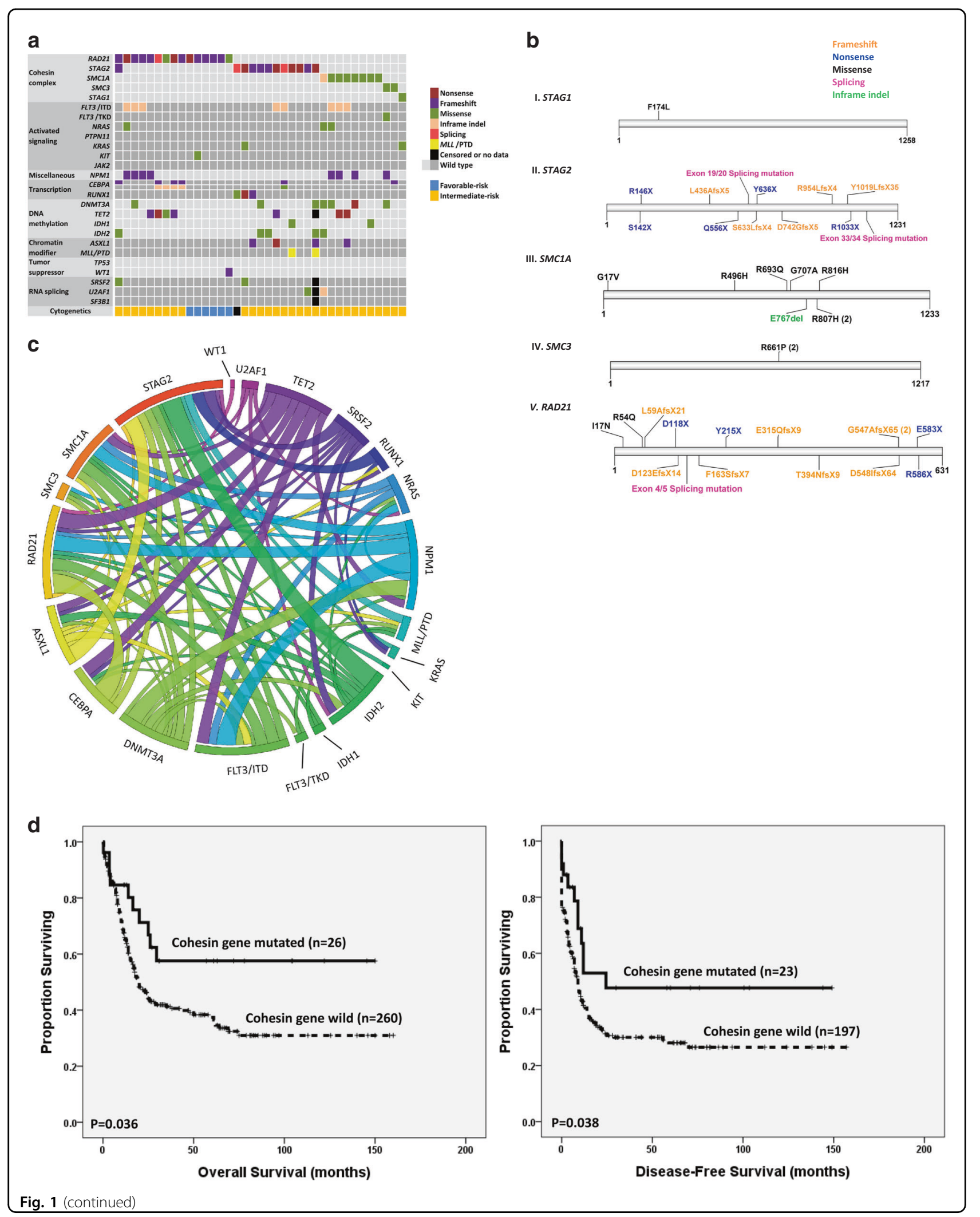




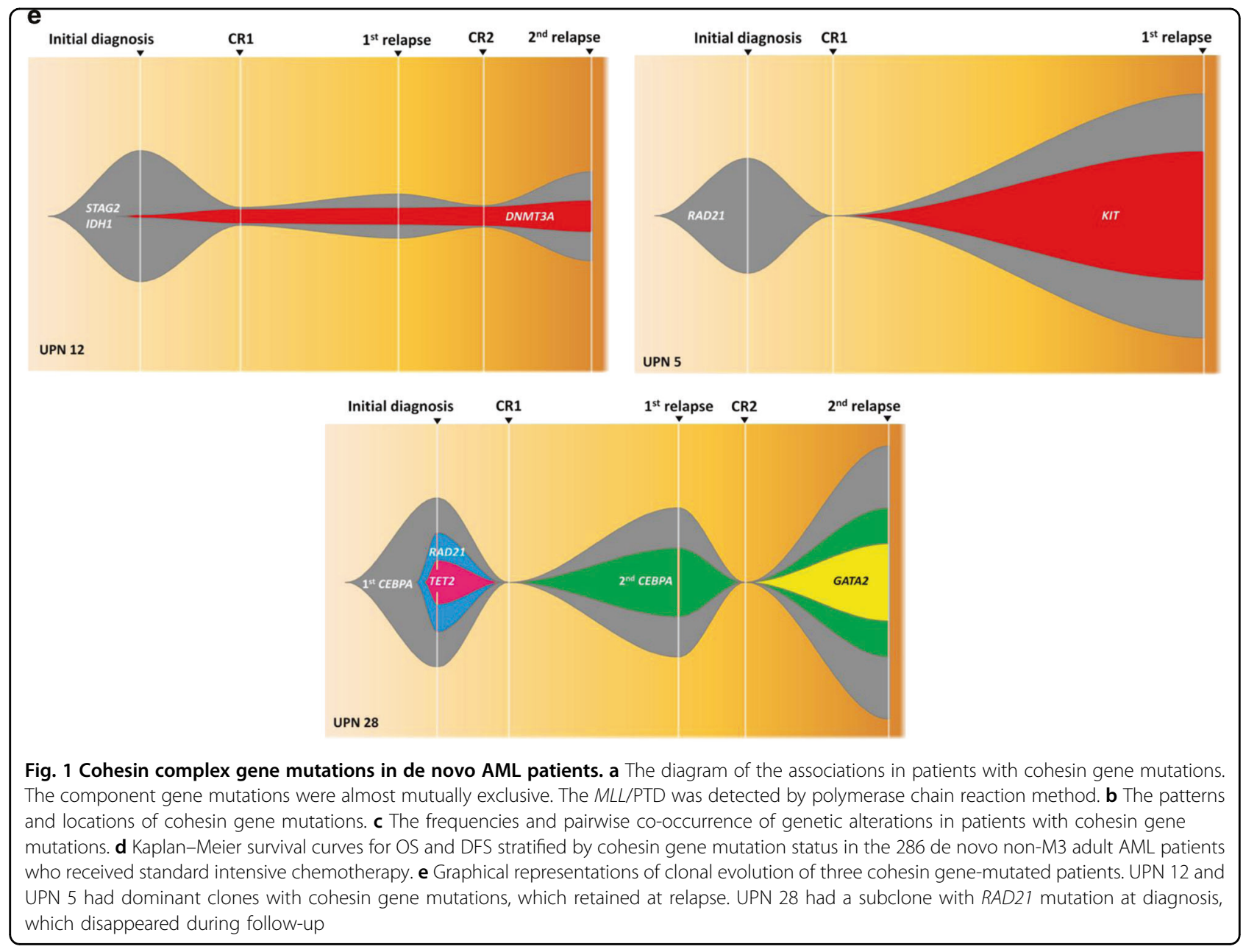

excluded from this study, none harbored a cohesin gene mutation ( $0 \%$ vs. $9.5 \%, P=0.059$, data not shown).

We screened mutations in 20 other genes, including FLT3, NPM1, CEBPA, RUNX1, ASXL1, IDH1,IDH2, TET2 ${ }^{9}$, DNMT3A, NRAS, KRAS, JAK2, KIT, PTPN11, SRSF2, U2AF1, SF3B1 ${ }^{10}, W T 1, T P 53$, and $M L L /$ PTD $^{11}$, to investigate the difference of the mutation profiles between cohesin gene-mutated and wild-type (WT) AML patients. Among the 37 patients with cohesin gene mutations, 30 (81.1\%) patients had at least one other gene mutation simultaneously. The most common concurrent molecular events in cohesin gene-mutated cohort were FLT3/ITD (21.6\%) and NPM1 mutations (21.6\%). None of the patients with cohesin gene mutations had TP53 mutations. Compared with other cohesin gene mutations, STAG2 mutations more frequently co-occurred with RUNX1 mutations $(27.3 \%$ vs. $0 \%, P=0.023)$ and tended to co-occur with $A S X L 1$ mutations $(25.0 \%$ vs. $4.0 \%, P=0.084)$, but less frequently with NPM1 mutations ( $0 \%$ vs. $32.0 \%, P=0.036$; Fig. 1a and Supplementary Table S2).
Until now, reports regarding prognostic relevance of cohesin gene mutations in AML are very limited. In this study, survival analyses were performed in the 286 (73.1\%) patients who received standard chemotherapy, including 26 cohesin gene-mutated and 260 WT patients. The complete remission (CR), induction death, and relapse rate were similar between these two groups (Supplementary Table S3). With a median follow-up time of 53.0 months (range, 0.1-160), patients with cohesin gene mutations had significantly longer overall survival (OS) and disease-free survival (DFS) than those without the mutation (not reached vs. $20.0 \pm 2.3$ months, $P=0.036$ and $24.5 \pm 0.0$ vs. $9.0 \pm 0.8$ months, $P=0.038$, respectively, Fig. 1d). The detail of univariate analysis for OS and DFS was shown in Supplementary Table S4.

In multivariate Cox proportional hazards regression analysis for OS and DFS, cohesin gene mutations were independent favorable factors for both OS and DFS (Table 1 ). These results were in contrast to the report of Thol et al. in which cohesin gene mutations had no significant implication on OS and DFS (OS hazard ratio (HR) 
Table 1 Multivariate analysis of the disease-free survival and overall survival

\begin{tabular}{|c|c|c|c|c|c|c|c|c|}
\hline \multirow[t]{3}{*}{ Variables } & \multicolumn{4}{|c|}{ Disease-free survival } & \multicolumn{4}{|c|}{ Overall survival } \\
\hline & \multirow[t]{2}{*}{ RR } & \multicolumn{2}{|l|}{$95 \% \mathrm{Cl}$} & \multirow[t]{2}{*}{$P$ value } & \multirow[t]{2}{*}{ RR } & \multirow{2}{*}{$\begin{array}{l}95 \% \mathrm{Cl} \\
\text { Lower }\end{array}$} & \multirow[b]{2}{*}{ Upper } & \multirow[t]{2}{*}{$P$ value } \\
\hline & & Lower & Upper & & & & & \\
\hline \multicolumn{9}{|l|}{ Total cohort $(n=286)$} \\
\hline $\operatorname{Age}^{\mathrm{a}}$ & 1.916 & 1.401 & 2.621 & $<0.001$ & 2.462 & 1.755 & 3.453 & $<0.001$ \\
\hline$W B C^{b}$ & 1.356 & 0.983 & 1.871 & 0.063 & 1.552 & 1.096 & 2.198 & 0.013 \\
\hline Karyotype $^{c}$ & 1.553 & 0.982 & 2.456 & 0.060 & 1.936 & 1.219 & 3.076 & 0.005 \\
\hline NPM1/FLT3-ITD & 0.260 & 0.121 & 0.560 & 0.001 & 0.240 & 0.097 & 0.592 & 0.002 \\
\hline CEBPA double mutations e & 0.504 & 0.301 & 0.844 & 0.009 & 0.352 & 0.182 & 0.678 & 0.002 \\
\hline RUNX1 $1^{\mathrm{e}}$ & 0.977 & 0.593 & 1.611 & 0.928 & 1.012 & 0.593 & 1.728 & 0.965 \\
\hline $\operatorname{ASXL1^{\mathrm {e}}}$ & 0.973 & 0.563 & 1.683 & 0.922 & 1.134 & 0.646 & 1.990 & 0.662 \\
\hline $\mathrm{IDH} 2^{\mathrm{e}}$ & 0.845 & 0.518 & 1.377 & 0.498 & 0.490 & 0.260 & 0.924 & 0.028 \\
\hline Cohesin gene $e^{e}$ & 0.487 & 0.256 & 0.926 & 0.028 & 0.489 & 0.242 & 0.992 & 0.047 \\
\hline $\mathrm{SF}^{\mathrm{e}}$ & 1.992 & 1.252 & 3.171 & 0.004 & 1.702 & 1.006 & 2.879 & 0.048 \\
\hline$T P 53^{\mathrm{e}}$ & 1.512 & 0.817 & 2.797 & 0.188 & 1.697 & 0.916 & 3.146 & 0.093 \\
\hline
\end{tabular}

$R R$ relative risk, $\mathrm{Cl}$ confidence interval, $S F$ splicing factor genes

${ }^{a}$ Age $>50$ years relative to age $\leq 50$ years (the reference)

${ }^{\text {b } W B C}>50,000 / \mu \mathrm{l}$ vs. $\leq 50,000 / \mu \mathrm{l}$

'Unfavorable cytogenetics vs. others

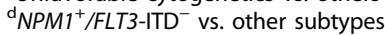

${ }^{\mathrm{e}}$ Mutated vs. wild type

$0.96, P=0.89$ and RFS HR $0.62, P=0.18$, respectively $)^{5}$. In that report, the incidence $(5.9 \%)$ of cohesin gene mutations in AML was relatively low, compared with that in other reports $(8.8-13.3 \%)^{4,6,7}$, and patients with secondary AML were included. Thota et al. reported that in patients with myelodysplastic syndrome (MDS) who survived more than 12 months, cohesin gene mutations were associated with a shorter survival (HR 2.1, $P=$ 0.017). The prognostic implication of cohesin gene mutations in primary AML patients $(n=101)$ was not analyzed in that report ${ }^{6}$. The reasons why the cohesin gene mutations have opposite prognostic impact on AML and MDS remain unknown. Similar findings have been found in SF3B1 mutation, which has a negative impact on de novo AML patients ${ }^{2,10}$, but a favorable impact on patients with $\mathrm{MDS}^{12}$.

In order to evaluate the dynamic changes of cohesin and co-occurring gene mutations, we serially analyzed 386 samples from 116 patients, including 19 with and 97 without cohesin gene mutations at diagnosis (Table 2), for 54 gene mutations involved in myeloid malignancies by TruSight Myeloid Panel (Illumina, San Diego, CA, USA). HiSeq platform (Illumina) was used for sequencing with a median reading depth of $12,000 \times$. Among the patients with cohesin gene mutations, 17 patients lost the original mutations at $\mathrm{CR}$, while the mutations remained detectable at CR in UPN 1 and 12
(Fig. 1e), although with lower allele frequencies. The disease subsequently relapsed in these two patients with rising mutant allele burdens indicating the presence of minimal residual disease. Most other concurrent mutations in the 19 patients studied disappeared at CR but DNMT3A mutations were detectable in four patients (UPN 12, 16, 20, and 22), in whom IDH1 (UPN 12), U2AF1 (UPN 20 in CR2), or NPM1 and NRAS mutations (UPN 22) also remained detectable at the same time. Two (UPN 28 and 37) of the eight cohesin-mutated patients who had paired samples at both diagnosis and relapse lost the original cohesin gene mutations (both in RAD21) during disease evolution. Graphical representations of clonal evolution in three representative patients were shown in Fig. 1e. Among the 97 patients without cohesin gene mutations at diagnosis, no one acquired the mutation at relapse, indicating that the mutations played little role in the progression of AML.

Furthermore, we applied Bradley-Terry model to evaluate the temporal order of gene mutations in cohesinmutated patients (Supplementary Fig. S1). Only samples with statistically significant and recurrent gene-gene pairwise precedence were included in the analysis. The STAG2 mutations occurred as an early event, while RAD21 and SMC1A mutations occurred relatively late. In comparison of gene mutations between secondary and de novo AML, Lindsley et al. defined STAG2 mutation as a 
Table 2 Sequential studies in the AML patients with cohesin gene mutations at diagnosis ${ }^{\mathrm{a}}$

\begin{tabular}{|c|c|c|c|c|c|}
\hline \multirow[t]{2}{*}{$U_{P N}^{a}$} & \multirow[t]{2}{*}{ Interval $^{\mathbf{b}}$ (months) } & \multirow[t]{2}{*}{ Disease status } & \multirow[t]{2}{*}{ Karyotype } & \multicolumn{2}{|l|}{ Mutations } \\
\hline & & & & Cohesin & Others \\
\hline \multirow[t]{3}{*}{1} & & Diagnosis & $\begin{array}{l}49, X Y, t(6 ; 11)(q 27 ; q 23),+8, \\
+9,+19\end{array}$ & STAG1 (F174L) & KRAS \\
\hline & 0.8 & CR1 & $46, X Y$ & STAG1 (F174L) & - \\
\hline & 2.0 & Relapse1 & $46, X Y$ & STAG1 (F174L) & - \\
\hline \multirow[t]{3}{*}{5} & & Diagnosis & $46, X Y, t(8 ; 21)(q 22 ; q 22)$ & RAD21 (Y215Ter) & - \\
\hline & 0.9 & CR1 & $46, X Y$ & - & - \\
\hline & 12.0 & Relapse1 & $46, X Y, t(8 ; 21)(q 22 ; q 22)$ & RAD21 (Y215Ter) & KIT \\
\hline \multirow[t]{2}{*}{6} & & Diagnosis & NM & STAG2 (exon 19/20 splicing) & $B C O R, B C O R L 1, C S F 3 R, R \cup N X 1$ \\
\hline & 1.0 & CR1 & $46, X Y$ & - & - \\
\hline \multirow[t]{2}{*}{9} & & Diagnosis & $46, X Y$ & $\begin{array}{l}\text { STAG2 (D742GfsTer5), RAD21 } \\
\text { (G547AfsTer65) }\end{array}$ & IDH2, SRSF2, CEBPA \\
\hline & 2.6 & CR1 & $46, X Y$ & - & - \\
\hline \multirow[t]{3}{*}{11} & & Diagnosis & $46, X Y$ & STAG2 (S633LfsTer4) & BCOR, DNMT3A, IDH2 \\
\hline & 14.0 & Relapse1 & $46, X Y$ & STAG2 (S633LfsTer4) & BCOR, DNMT3A, IDH2 \\
\hline & 6.8 & CR2 & $46, X Y$ & - & - \\
\hline \multirow[t]{5}{*}{12} & & Diagnosis & $46, X Y$ & STAG2 (Q556Ter) & IDHI, DNMT3A \\
\hline & 1.1 & CR1 & ND & STAG2 (Q556Ter) & IDHI, DNMT3A \\
\hline & 8.2 & Relapse1 & ND & STAG2 (Q556Ter) & IDH1, DNMT3A \\
\hline & 4.4 & CR2 & ND & STAG2 (Q556Ter) & IDHI, DNMT3A \\
\hline & 2.3 & Relapse 2 & ND & STAG2 (Q556Ter) & IDH1, DNMT3A \\
\hline \multirow[t]{2}{*}{16} & & Diagnosis & $46, X Y$ & SMC3 (R661P) & DNMT3A, FLT3/TKD, NPM1 \\
\hline & 1.3 & CR1 & $46, X Y$ & - & DNMT3A \\
\hline \multirow[t]{2}{*}{18} & & Diagnosis & $46, X X$ & SMC1A (R496H) & FLT3/ITD, NPM1, TET2 \\
\hline & 1.0 & CR1 & ND & - & - \\
\hline \multirow[t]{2}{*}{19} & & Diagnosis & $46, X Y$ & SMC1A (G707A) & - \\
\hline & 10.0 & CR1 & $46, X Y$ & - & - \\
\hline \multirow[t]{5}{*}{20} & & Diagnosis & $47, X Y,+8$ & SMC1A (E767del) & DNMT3A, IDH2, NRAS, U2AF1 \\
\hline & 1.7 & CR1 & $47, X Y$ & - & DNMT3A \\
\hline & 13.8 & Relapse1 & $48, X Y,+8,+15$ & SMC1A (E767del) & DNMT3A, IDH2, NRAS, U2AF1 \\
\hline & 2.1 & CR2 & ND & - & DNMT3A, U2AF1 \\
\hline & 8.1 & Relapse2 & $48, X Y,+X,+15$ & SMC1A (E767del) & $\begin{array}{l}\text { DNMT3A, IDH2, NRAS, U2AF1, } \\
\text { RUNX1, CUX1 }\end{array}$ \\
\hline \multirow[t]{4}{*}{22} & & Diagnosis & $45, X,-Y$ & SMC1A (R693Q) & DNMT3A, NPM1, NRAS, FLT3/ITD \\
\hline & 0.9 & CR1 & NM & - & DNMT3A, NPM1, NRAS \\
\hline & 23.9 & Relapse1 & $45, X,-Y$ & SMC1A (R693Q) & DNMT3A, NPM1, FLT3/ITD \\
\hline & 1.2 & CR2 & $46, X Y$ & - & - \\
\hline \multirow[t]{2}{*}{23} & & Diagnosis & $46, X Y$ & SMC1A (G17V) & $\mid \mathrm{DH} 1$ \\
\hline & 1.2 & CR1 & $46, X Y$ & - & - \\
\hline 24 & & Diagnosis & $45, X,-Y$ & SMC1A (R816H) & NPM1, DNMT3A \\
\hline
\end{tabular}


Table 2 continued

\begin{tabular}{|c|c|c|c|c|c|}
\hline \multirow[t]{2}{*}{ UPN $^{\mathrm{a}}$} & \multirow[t]{2}{*}{ Interval ${ }^{\mathbf{b}}$ (months) } & \multirow[t]{2}{*}{ Disease status } & \multirow[t]{2}{*}{ Karyotype } & \multicolumn{2}{|l|}{ Mutations } \\
\hline & & & & Cohesin & Others \\
\hline & 1.7 & CR1 & $46, X Y$ & - & - \\
\hline 27 & & Diagnosis & $46, X Y$ & RAD21 (D118Ter) & CEBPA, GATA2, TET2 \\
\hline & 1.0 & CR1 & ND & - & - \\
\hline 28 & & Diagnosis & $46, X Y$ & RAD21 (I17Asn) & CEBPA $^{\mathrm{C}}$, TET2 \\
\hline & 1.0 & CR1 & ND & - & - \\
\hline & 9.1 & Relapse1 & ND & - & CEBPA $^{\mathrm{C}}$ \\
\hline & 2.8 & CR2 & ND & - & - \\
\hline & 6.0 & Relapse2 & ND & - & CEBPA ${ }^{\circ}$, GATA2 \\
\hline 29 & & Diagnosis & $46, X Y$ & RAD21 (exon 4/5 splicing) & CEBPA, CSF3R, TET2 \\
\hline & 1.5 & CR1 & ND & - & - \\
\hline 32 & & Diagnosis & $46, X X$ & RAD21 (R54Q) & PHF6, WT1, ETV6 \\
\hline & 84.7 & CR1 & $46, X X$ & - & - \\
\hline 36 & & Diagnosis & $46, X X, t(8 ; 21)$ & RAD21 (D548IfsTer64) & PTPN11, KDM6A \\
\hline & 5.0 & CR1 & $46, X X$ & - & - \\
\hline 37 & & Diagnosis & 46, XX, del(11)(q14q23) & RAD21 (T394NfsTer9) & CEBPA \\
\hline & 1.0 & CR1 & $46, X X$ & - & - \\
\hline & 5.0 & Relapse1 & $46, X X$ & - & - \\
\hline
\end{tabular}

UPN unique patient number, $C R$ complete remission, $N D$ not done, $N M$ no mitosis

${ }^{a}$ The data of serial studies in other 97 patients who did not have cohesin gene mutation at diagnosis were not shown in this table. None of them acquired a cohesin gene mutation at relapse

Interval between the two successive studies

'UPN 28 had one CEBPA mutation at diagnosis (K313dup) but had two mutations at both relapse 1 and relapse 2 (K313dup and 168RfsTer39)

secondary-type mutation because it was $95 \%$ specific for secondary $\mathrm{AML}^{2}$. However, other studies suggested that STAG2 mutation might serve as an early event in leukemogenesis in $\mathrm{AML}^{3,5,6,13,14}$.

We further profiled genome-wide mRNA expression in 10 cohesin-mutated and $163 \mathrm{WT}$ patients to explore the molecular mechanisms underlying cohesin gene mutations. One hundred and sixty-two differentially expressed genes were identified between the cohesin-mutated and WT AML ( $>1.5$-fold change and $t$-test $P<0.05$, Supplementary Fig. S2 and Supplementary Table S5). Ingenuity Pathway Analysis (IPA, Ingenuity Systems, Redwood City, CA, USA) and Gene Set Enrichment Analysis (GSEA) revealed that these genes were significantly associated with differentiation of blood cells, proliferation of blood cells, apoptosis, and cell death of blood cells (Supplementary Table S6 and Supplementary Fig. S3). Besides, a network constructed by IPA showed ERK1/2 was a hub gene among the differentially expressed genes, implying involvement of this multifunctional kinase in cohesin gene mutation-driven signaling (Supplementary Fig. S4A). ERK1/2 still played a central role in the networks constructed by the differentially expressed genes between STAG2-mutated and WT patients, and RAD21mutated and WT patients (Supplemental Figs. S4B and S4C).

In conclusion, our study showed that cohesin gene mutations were recurrent in de novo AML and had favorable impacts on both OS and DFS. Further, cohesin gene mutations were strongly associated with the biological function related to proliferation and differentiation of blood cells. Sequential analyses showed cohesin gene mutations might be lost during disease evolution in de novo AML patients, but none of the patients without the mutation acquired a novel one during the clinical course. Further prospective studies with larger cohorts are warranted to confirm our findings.

\section{Acknowledgments}

This work was partially sponsored by grants MOST 100-2628-B-002-003-MY3, 103-2628-B-002-008-MY3, 103-2923-B-002-001, MOST 102-2325-B-002-028, $103-$ 2314-B-002-130-MY3, 103-2314-B-002-131MY3, and 104-2314-B-002-128-MY4 from the Ministry of Science and Technology (Taiwan), National Taiwan University Hospital-National Taiwan University joint research grant (UN103051), Far Eastern Hospital and NTUH joint grant 105-FTN24, and MOHW 105TDU-B-211-134004 from the Ministry of Health and Welfare (Taiwan), NTUH 
102P06, from the Department of Medical Research, National Taiwan University Hospital, and Taiwan Health Foundation. We would like to acknowledge the service provided by the DNA Sequencing Core of the First Core Laboratory, National Taiwan University College of Medicine.

\section{Author details}

'Division of Hematology, Department of Internal Medicine, National Taiwan University Hospital, Taipei, Taiwan. ${ }^{2}$ Tai-Cheng Stem Cell Therapy Center, National Taiwan University, Taipei, Taiwan. ${ }^{3}$ Genome and Systems Biology Degree Program, National Taiwan University, Taipei, Taiwan. ${ }^{4}$ Graduate Institute of Oncology, National Taiwan University, Taipei, Taiwan. ${ }^{5}$ Graduate Institute of Biomedical Electronics and Bioinformatics, National Taiwan University, Taipei, Taiwan. ${ }^{6}$ Department of Laboratory Medicine, National Taiwan University Hospital, Taipei, Taiwan. ${ }^{7}$ Graduate Institute of Clinical Medicine, National Taiwan University, Taipei, Taiwan. ${ }^{8}$ Biostatistics Consulting Laboratory, School of Nursing and Center of General Education, National Taipei University of Nursing and Health Sciences, Taipei, Taiwan. ${ }^{9}$ Department of Pathology, National Taiwan University Hospital, Taipei, Taiwan. ${ }^{10}$ Department of Clinical Laboratory Sciences and Medical Biotechnology, College of Medicine, National Taiwan University, Taipei, Taiwan. ${ }^{11}$ Bioinformatics and Biostatistics Core, Center of Genomic Medicine, National Taiwan University, Taipei, Taiwan

\section{Author contributions}

C.-H.T. was responsible for data management and interpretation, mutation analysis, statistical analysis, and manuscript writing; H.-A.H. was responsible for study design and plan, data management and interpretation, mutation analysis, statistical analysis, and manuscript writing; Y.-C.C. and E.Y.C. were responsible for network analysis; C.-Y.L. was responsible for statistical analysis and interpretation of the statistical findings; Y.-Y.K., T.Y.L., and L.-I.L. were responsible for mutation analysis and interpretation; J.-L.T., C.-C.L., M.Y., C.-C.L., S.-Y.H., B.-S.K., S.-C.H., C.-T.L., S.-J.W., C.-Y.C., and W.T. contributed patient samples and clinical data; M.-H.T., C.-Y. L., and M.-C.L. performed the gene mutation and chromosomal studies; W.-C.C. and H.-F.T. designed and coordinated the study over the entire period and wrote the manuscript.

\section{Competing interests}

The authors declare that they have no competing interests.

\section{Publisher's note}

Springer Nature remains neutral with regard to jurisdictional claims in published maps and institutional affiliations.

\section{Supplementary information}

The online version of this article (https://doi.org/10.1038/s41408-017-0022-y) contains supplementary material.

Received: 25 August 2017 Revised: 8 October 2017 Accepted: 12 October 2017

Published online: 29 December 2017

\section{References}

1. Duployez, N. et al. Comprehensive mutational profiling of core binding factor acute myeloid leukemia. Blood 127, 2451-2459 (2016).

2. Lindsley, R. C. et al. Acute myeloid leukemia ontogeny is defined by distinct somatic mutations. Blood 125, 1367-1376 (2015).

3. Papaemmanuil, E. et al. Genomic Classification and Prognosis in Acute Myeloid Leukemia. N. Engl. J. Med. 374, 2209-2221 (2016).

4. Cancer Genome Atlas Research, N. et al. Genomic and epigenomic landscapes of adult de novo acute myeloid leukemia. N. Engl. J. Med. 368, 2059-2074 (2013).

5. Thol, F. et al. Mutations in the cohesin complex in acute myeloid leukemia: clinical and prognostic implications. Blood 123, 914-920 (2014).

6. Thota, S. et al. Genetic alterations of the cohesin complex genes in myeloid malignancies. Blood 124, 1790-1798 (2014).

7. Kon, A. et al. Recurrent mutations in multiple components of the cohesin complex in myeloid neoplasms. Nat. Genet. 45, 1232-1237 (2013).

8. Faber, Z. J. et al. The genomic landscape of core-binding factor acute myeloid leukemias. Nat. Genet. 48, 1551-1556 (2016).

9. Chou, W. C. et al. TET2 mutation is an unfavorable prognostic factor in acute myeloid leukemia patients with intermediate-risk cytogenetics. Blood 118, 3803-3810 (2011).

10. Hou, H. A. et al. Splicing factor mutations predict poor prognosis in patients with de novo acute myeloid leukemia. Oncotarget 7, 9084-9101 (2016).

11. Shiah, H. S. et al. Clinical and biological implications of partial tandem duplication of the MLL gene in acute myeloid leukemia without chromosomal abnormalities at 11q23. Leukemia 16, 196-202 (2002).

12. Malcovati, L. et al. SF3B1 mutation identifies a distinct subset of myelodysplastic syndrome with ring sideroblasts. Blood 126, 233-241 (2015).

13. Mazumdar, C. et al. Leukemia-Associated Cohesin Mutants Dominantly Enforce Stem Cell Programs and Impair Human Hematopoietic Progenitor Differentiation. Cell. Stem. Cell. 17, 675-688 (2015).

14. Mullenders, J. et al. Cohesin loss alters adult hematopoietic stem cell homeostasis, leading to myeloproliferative neoplasms. J. Exp. Med. 212, 1833-1850 (2015). 